\title{
Corela
}

Cognition, représentation, langage

HS-7 | 2010

Espace, Préposition, Cognition

\section{Claude Vandeloise : bibliographie des travaux / bibliography of his works}

\section{Michel Aurnague}

\section{(2) OpenEdition}

1 Journals

\section{Édition électronique}

URL : http://journals.openedition.org/corela/1755

DOI : $10.4000 /$ corela. 1755

ISSN : 1638-573X

Éditeur

Cercle linguistique du Centre et de l'Ouest - CerLICO

\section{Référence électronique}

Michel Aurnague, "Claude Vandeloise : bibliographie des travaux / bibliography of his works », Corela [En ligne], HS-7 | 2010, mis en ligne le 31 mai 2010, consulté le 14 septembre 2020. URL : http:// journals.openedition.org/corela/1755

Ce document a été généré automatiquement le 14 septembre 2020.

\section{(c) (i) (2)(2)}

Corela - cognition, représentation, langage est mis à disposition selon les termes de la licence Creative Commons Attribution - Pas d'Utilisation Commerciale - Partage dans les Mêmes Conditions 4.0 International. 


\section{Claude Vandeloise : bibliographie des travaux / bibliography of his works}

Michel Aurnague

\section{Ouvrages / Books}

1 Vandeloise, C. (1986a). L'espace en français : sémantique des prépositions spatiales. Paris: Editions du Seuil (Travaux Linguistiques).

2 Vandeloise, C. (1991a). Spatial prepositions: a case study in French (traduit par / translated by A.R. Bosch). Chicago, IL: The University of Chicago Press.

3 Vandeloise, C. (2001). Aristote et le lexique de l'espace : rencontres entre la physique grecque et la linguistique cognitive. Stanford, CA: CSLI (Langage et Esprit).

4 Vandeloise, C. \& F.A. Anselmo (2002). Introduction to French linguistics. Munich: Lincom (Studies in Romance Linguistics 21).

5 Vandeloise, C. (2004a). La dimension en français : de l'espace à la matière. Paris: Hermes.

6 Vandeloise, C. (2006a). De la distribution à la cognition. Paris: L'Harmattan (Sémantiques).

\section{Edition d'ouvrages et de numéros de revues / Edited books and journal issues}

Vandeloise, C. (1987a). L'expression du mouvement. Langue Française, 76.

Vandeloise, C. (1991b). Sémantique Cognitive. Communications, 53.

9 Vandeloise, C. (1993a). La couleur des prépositions. Langages, 110.

10 Vandeloise, C. (2003a). Langues et cognition. Paris: Hermes (Traité des Sciences Cognitives). 


\section{Contributions à des revues et à des ouvrages / Contributions to journals and books}

11 Vandeloise, C. (1985a). Au-delà des descriptions géométriques et logiques de l'espace : une description fonctionnelle. Linguisticae Investigationes, 9(1): 109-129.

Vandeloise, C. (1985b). La préposition dans et la relation contenant/contenu. Leuvense Bijdragen, 74(2): 193-208.

Vandeloise, C. (1985c). Les prépositions sur/sous et la relation porteur/porté. Leuvense Bijdragen, 74(4): 457-481.

Vandeloise, C. (1985d). Les prépositions avant/après et l'ordre dans la rencontre potentielle. Recherches Linguistiques de Vincennes, 13: 110-129.

Vandeloise, C. (1986b). L'avant/l'arrière et le devant/le derrière. Revue Québecoise de Linguistique, 16(1): 281-308.

16 Vandeloise, C. (1987b). La préposition à et le principe d'anticipation. Langue Française, 76: 77-110.

17 Vandeloise, C. (1987c). Complex primitives in language acquisition. Belgian Journal of Linguistics, 2 (Perspectives on child language, A. De Houwer \& S. Gilles (Eds.)): 11-36.

18 Vandeloise, C. (1987d). Les prépositions devant/derrière, l'orientation générale et l'accès à la perception. Le Français Moderne, 55(1-2): 1-22.

19 Vandeloise, C. (1988a). Lenght, width and potential passing. In B. Rudzka-Ostyn (Ed.), Topics in cognitive grammar. Amsterdam: John Benjamins (Current Issues in Linguistic Theory 50), 403-427.

20 Vandeloise, C. (1988b). Les usages spatiaux statiques de la preposition à. Cahiers de Lexicologie, 53: 119-148.

21 Vandeloise, C. (1989). L'expression linguistique de la relation de suspension. Cahiers de Lexicologie, 55: 111-148.

22 Vandeloise, C. (1990a). Representation, prototypes and centrality. In S.L. Tsohatzidis (Ed.), Meanings and prototypes: studies in linguistic categorization. Londres / London \& New York: Routledge, 403-437.

23 Vandeloise, C. (1990b). Chronique. Règles ou listes : l'arbitrage de la morphologie. Le Français Moderne, 58(3-4): 224-234.

24 Vandeloise, C. (1990c). Les frontières entre les prépositions sur et à. Cahiers de Grammaire, 15: 157-184. Vandeloise, C. (1991c). Autonomie du langage et cognition. Communications, 53: 69-103. Vandeloise, C. (1992a). Quand le silence prend la parole. In L. Tasmowski \& A. ZribiHertz (Eds.), De la musique à la linguistique, Hommages à Nicolas Ruwet. Gand / Ghent: Communication \& Cognition, 634-640. Vandeloise, C. (1992b). Une catégorie lexicale et le défi de l'art. In D. Andler, P. Jacob, J. Proust, F. Récanati \& D. Sperber (Eds.), Philosophie et cognition. Liège: Mardaga, 255-263.

28 Vandeloise, C. (1992c). Objets en miroir et objets manufacturés : une hypothèse. Cahiers de Praxématique, 18 (Langage, cognition, expérience pratique, J.M. Barberis \& F. MadrayLesigne (Eds.)): 75-89. 
Vandeloise, C. (1992d). Les adjectifs de dimension: une analyse modulaire ou une analyse globale? Cahiers de Lexicologie, 61: 85-110.

Vandeloise, C. (1993b). La préposition à pâlit-elle derrière toucher? Langages, 110: 107-127.

Vandeloise, C. (1993c). The role of resistance in the meaning of thickness. LeuvenseBijdragen, 82(1): 29-47.

Vandeloise, C. (1993d). Espace et motivation. Faits de Langue, 1 (Iconicité et motivation, L. Danon-Boileau (Ed.)): 181-188.

Vandeloise, C. (1993e). Les analyses de la préposition dans : faits linguistiques et effets méthodologiques. Lexique, 11 (Les prépositions : méthodes d'analyse, A.M. Berthonneau \& P. Cadiot (Eds)): 15-40.

Vandeloise, C. (1994a). Methodology and analyses of the preposition in. Cognitive Linguistics, 5(2): 157-184.

Vandeloise, C. (1994b). Chronique. La catégorisation en sémantique cognitive. Le Français Moderne, 62(1): 79-98.

Vandeloise, C. (1995a). Cognitive linguistics and prototypes. In T. Sebeok \& J. UmikerSebeok (Eds.), Advances in visual semiotics: the semiotic web 1992-93. Berlin: Mouton de Gruyter (Approaches to Semiotics 118), 423-442.

Vandeloise, C. (1995b). De la matière à l'espace: la préposition dans. Cahiers de Grammaire, 20: 123-145.

Vandeloise, C. (1995c). Le rôle de la place dans la définition de la préposition dans : vers une ontologie fantomatique. In P. Amsili, M. Borillo \& L. Vieu (Eds.), Time, Space and Movement: meaning and knowledge in the sensible world, Workshop Notes of the $5^{\text {th }}$ International Workshop TSM'95. Toulouse: LRC, 165-179 (Part A).

9 Vandeloise, C. (1996a). Touching: a minimal transmission of energy. In E. Casad (Ed.), Cognitive linguistics in the redwoods: the expansion of a new paradigm in linguistics. Berlin: Mouton de Gruyter (Cognitive Linguistic Research 6), 541-566.

40 Vandeloise, C. (1996b). La méronomie, l'inclusion topologique et la préposition dans. Faits de Langue, 7 (La relation d'appartenance, L. Danon-Boileau \& M.A. Morel (Eds.)): 81-90.

41 Vandeloise, C. (1998). Les domaines des prépositions avant/après. Verbum, 20(4) (Prépositions et métaphore, W. De Mulder \& N. Flaux (Eds.)): 383-395.

2 Vandeloise, C. (1999a). Une place pour chaque chose et chaque chose à sa place. In M. Plénat, M. Aurnague, A. Condamines, J.P. Maurel, C. Molinier \& C. Muller (Eds.), L'emprise du sens : structures linguistiques et interprétations, Mélanges offerts à Andrée Borillo. Amsterdam: Rodopi (Faux Titre 174), 303-321.

Vandeloise, C. (1999b). Quand dans quitte l'espace pour le temps. Revue de Sémantique et Pragmatique, 6 (Approches sémantiques des prépositions, P. Cadiot \& A. Ibrahim (Eds.)): 145-162.

44 Vandeloise, C. (2000a). Plus de clarté dans la relation porteur/porté. In M. Coene, W. De Mulder, P. Dendale \& Y. D’Hulst (Eds.), Traiani Augusti vestigia pressa sequamur, Studia linguistica in honorem Lilianae Tasmowski. Padoue / Padova: Unipress, 753-770.

Vandeloise, C. (2000b). Verbes de changement, de transformation et de génération. Cahiers de Lexicologie, 77: 117-136. 


\section{dans la langue et la métal
Linguistiques 27), 121-150.}

Harel (Eds.), Lieux propices : l'énonciation des lieux/le lieu de l'énonciation, dans les contextes francophones interculturels. Laval: Presses de l'Université de Laval (Intercultures), 295-310.

Vandeloise, C. (2002a). La prédication de la matière entre prédicats nominaux et prédicats adjectivaux. Le Français Moderne, 70(1): 20-44.

Vandeloise, C. (2003b). Diversité linguistique et cognition. In C. Vandeloise (Ed.), Langues et cognition. Paris: Hermes (Traité des Sciences Cognitives), 19-58.

Vandeloise, C. (2003c). Acquisition des termes spatiaux et relativisme linguistique. In C. Vandeloise (Ed.), Langues et cognition. Paris: Hermes (Traité des Sciences Cognitives), 279-301.

Vandeloise, C. (2003d). Containment, support and linguistic relativity. In H. Cuyckens, R. Dirven \& J. Taylor (Eds.), Cognitive approaches to lexical linguistics. Berlin: Mouton de Gruyter (Cognitive Linguistic Research 23), 393-425.

Vandeloise, C. (2003e). Les usages spatiaux de la préposition contre. In P. Péroz (Ed.), Contre, identité sémantique et variation catégorielle. Metz: CELTED/Université de Metz (Recherches Linguistiques 26), 15-43. J.M. Fortis (Ed.)): 89-109. (Ed.), From perception to meaning: schemas in cognitive linguistics. Berlin: Mouton de Gruyter (Cognitive Linguistic Research 29), 343-366. perception, categorization, and development. Oxford: Oxford University Press (Explorations in Language and Space 2), 219-232.

Vandeloise, C. (2005b). L'évaluation globale des dimensions : les adjectifs gros, grand et long. Cahiers de Lexicologie, 87: 79-95.

Vandeloise, C. (2005c). Family resemblances and the structure of spatial relationships. Corela, 3(2): http://edel.univ-poitiers.fr/corela/document.php?id=726

Vandeloise, C. (2006b). Are there spatial prepositions? In M. Hickmann \& S. Robert (Eds.), Space in languages: linguistic systems and cognitive categories. Amsterdam: John Benjamins (Typological Studies in Language 66), 139-154.

Vandeloise, C. (2006c). Vivre sans article partitif. Le Français Moderne, 74(2): 141-158.

Vandeloise, C. (2007a). A taxonomy of basic natural entities. In M. Aurnague, M. Hickmann \& L. Vieu (Eds.), The categorization of spatial entities in language and cognition. Amsterdam: John Benjamins (Human Cognitive Processing 20), 35-52.

Vandeloise, C. (2007b). Le verbe aller : l'affranchissement du contexte d'énonciation immédiat. Journal of French Language Studies, 17(3): 343-359. 

cognition and space: the state of the art and newdirections. Londres / London: Equinox (Advances in Cognitive Linguistics), 171-192.

\section{Autres / Others}

Vandeloise, C. (1979). Les termes de dimension en français. Mémoire de Maîtrise / Master's dissertation, Université de Vincennes.

Vandeloise, C. (1984). Description of space in French. Thèse de Doctorat / PhD dissertation, University of California, San Diego. Duisburg: LAUTD.

Vandeloise, C. (1985e). Représentation linguistique du mouvement et de l'espace. Thèse de Doctorat / PhD dissertation, Ecole des Hautes Etudes en Sciences Sociales, Paris.

Vandeloise, C. (1992e). Structure of lexical categories and family resemblances. Duisburg: LAUD (Paper 325).

Vandeloise, C. (1995d). Les rôles de la matière dans la prédication. Carnets de Grammaire, 5. Toulouse: rapport ERSS report.

Vandeloise, C. (2002b). Relativité linguistique et cognition. Carnets de Grammaire, 9. Toulouse: rapport ERSS report.

Vandeloise, C. (2008). Three basic prepositions in French and in English: a comparison. Carnets de Grammaire, 19. Toulouse: rapport CLLE-ERSS report.

\section{Comptes rendus / Reviews (aperçu / survey)}

G. Fauconnier, “Espaces mentaux”. Le Français Moderne, 54(1-2), 1986: 105-108

A.M. Diller, "La pragmatique des questions et des réponses". Le Français Moderne, 54(1-2), 1986: 108-109.

R. Savary, "Ordre langagier, champ spatial et emplois figurés". Le Français Moderne, 55(1-2), 1987: 130-134

D. Hofstadter, "Gödel, Escher, Bach : les brins d'une guirlande éternelle". Le Français Moderne, 55(1-2), 1987: 134-138.

B. de Cornulier, "Effets de sens". Le Français Moderne, 55(3-4), 1987: 243-246.

G. Lakoff \& M. Johnson, "Les métaphores dans la vie quotidienne". Le Français Moderne, 55(3-4), 1987: 246-250.

J. Haiman (Ed.), "Iconicity in syntax”. Le Français Moderne, 55(3-4), 1987: 251-256.

C. Habel, M. Herweg \& K. Rehkämper (Eds.), "Raumkonzepte in verstehenprozessen: interdisziplinäre beiträge zu sprache und raum". Language, 67(1), 1991: 179-181.

J. Calvo Pérez \& C. Hernández Sacristán (Eds.), "Perceptual and topological criteria in linguistic description”. Le Français Moderne, 60(1), 1992: 106-107.

G. Kleiber, "Sémantique du prototype : catégories et sens lexical". The French Review, 66(3), 1993: 515-516.

N. Ruwet, “Syntax and human experience”. Cognitive Linguistics, 4(2), 1993: 196-201. 
81 J. Cervoni, “La préposition : étude sémantique et pragmatique”. Romance Philology, 48(1), 1994: 35-38.

\section{AUTEUR}

\section{MICHEL AURNAGUE}

Cognition, Langues, Langage, Ergonomie (CLLE-ERSS, UMR 5263) / CNRS \& Université de Toulouse-Le Mirail 